\title{
Revisiting G-CSF Support for Hematologic Recovery after Autologous Transplantation in AML Patients
}

\author{
Oberson J S', Novak $\mathbf{U}^{1}$, Taleghani BM², \\ Baerlocher $\mathbf{G M}^{2}$, Seipel $\mathrm{K}^{3}$, Mueller $\mathrm{BU}^{3}$, \\ Leibundgut $\mathrm{K}^{4}$, Zimmerli $\mathrm{S}^{\mathbf{5}}$ and Pabst $\mathrm{T}^{1 *}$ \\ ${ }^{1}$ Department of Medical Oncology, University Hospital \\ Bern, Switzerland \\ ${ }^{2}$ Department of Hematology, University Hospital Bern, \\ Switzerland \\ ${ }^{3}$ Department of Clinical Research, University Hospital \\ Bern, Switzerland \\ ${ }^{4}$ Department of Pediatric Hemato-Oncology, University \\ Hospital Bern, Switzerland \\ ${ }^{5}$ Department of Infectious Diseases, University Hospital \\ Bern, Switzerland
}

*Correspondling author: Thomas Pabst, Department of Medical Oncology, University Hospital Bern, 3010 Berne, Switzerland

Received: March 06, 2017; Accepted: April 06, 2017; Published: April 26, 2017

\begin{abstract}
In acute myeloid leukemia (AML) patients, using granulocyte colonystimulating factor (G-CSF) to support hematologic recovery in induction and consolidation treatment reduces the number of febrile episodes and the duration of neutropenia and hospitalization. However, the benefit and safety of administering G-CSF to enhance hematologic recovery in AML patients after autologous stem cell transplantation (ASCT) have not been reported so far. At our center, it was our policy to administer G-CSF after ASCT in all AML patients. In June 2015, increasing economic pressure prompted us to omit G-CSF after ASCT. In this retrospective study, we assessed the effects of changing our strategy from applying G-CSF for hematologic recovery after ASCT (in 103 AML patients) to omitting G-CSF (12 patients). We found that administering G-CSF shortened the median duration until neutrophil recovery was $>0.5 \mathrm{G} / \mathrm{l}$ after ASCT by four days $(P=.0001)$, and patients with $\mathrm{G}-\mathrm{CSF}$ tended to have fewer bacteremias (38.3\% versus 66.6\%; $P=.0654$ ). The median duration of hospitalization was two days longer in patients without G-CSF support (25 versus 23 days; $P=.0603$ ). According to the Swiss in-patient reimbursement system, the shorter hospitalization of $+\mathrm{G}-\mathrm{CSF}$ patients resulted in decreased total costs per patient of $3305 \mathrm{CHF}$ (48 Mio U of G-CSF), and $3367 \mathrm{CHF}$ (30 Mio $U)$. Finally, no differences were observed in disease free $(P=.0938)$ and overall survival $(P=.7999)$ rates between $+\mathrm{G}-\mathrm{CSF}$ versus $-\mathrm{G}-\mathrm{CSF}$ patients. Our data suggest that G-CSF support after ASCT is safe and associated with shorter time until neutrophil recovery, fewer bacteremia episodes, shorter hospitalization, and lower costs.
\end{abstract}

Keywords: Autologous; Transplant; AML; Leukemia; Recovery; Prognosis; Survival; Granulocyte-colony Stimulating factor; G-CSF; Consolidation

\section{Introduction}

Two cycles of induction chemotherapy yield complete remission (CR1) in up to $80 \%$ of young patients with newly diagnosed acute myeloid leukemia (AML) [1]. However, without subsequent treatment most patients will eventually relapse within few months. Consequently, they are offered consolidation treatment in CR1 with either conventional chemotherapy, autologous or allogeneic transplantation [2-4]. Among these options, high-dose chemotherapy (HDCT) with autologous stem cell transplantation (ASCT) can be applied to consolidate CR1 in AML patients who have favorable or intermediate risk cytogenetic and molecular abnormalities [5-8].

Despite autologous stem cell support, HDCT inevitably causes transient, but relevant cytopenias, with neutropenia exposing patients to the risk of infections, morbidity, and, rarely, a fatal outcome. Thus, shortening the duration of neutropenia with granulocytecolony stimulating factor (G-CSF) after ASCT is expected to reduce infectious complications [9]. In fact, G-CSF given to support hematologic recovery following induction chemotherapy in AML patients has been shown to reduce the duration of neutropenia, the number of febrile episodes, the duration of hospitalization and the requirement for parenteral antibiotics [10-13]. In contrast, it is of no benefit if given together with chemotherapy as a priming strategy to improve survival rates [14].
The situation in AML-as compared to lymphomas or myeloma -is complicated by the fact that myeloid blasts usually express receptors for hematopoietic growth factors such as G-CSF and granulocyte macrophage-colony stimulating factor (GM-CSF). In AML patients, treatment with myeloid growth factors has therefore been hampered by concerns on potential stimulation of leukemic cell growth. Consequently, randomized trials have analyzed whether the use of myeloid growth factors can reduce the duration of chemotherapyinduced neutropenia in AML patients without compromising antileukemic treatment. However, no consistent detrimental effects on survival have been reported with myeloid growth factors given to promote hematologic recovery following induction chemotherapy or after consolidation chemotherapy [10-13]. In contrast, the use of myeloid growth factors in AML patients to enhance hematologic recovery after HDCT with ASCT has not been similarly studied. In fact, it is largely unknown whether applying myeloid growth factors are safe after ASCT in AML patients.

At our center, it was our policy to support hematologic recovery with G-CSF after ASCT in AML patients. In June 2015, however, increasing economic pressure prompted us to omit G-CSF after ASCT thereby saving the costs of G-CSF. However, the introduction of biosimilar G-CSF compounds forced prizes for G-CSF to plummet and largely eliminated the cost argument in the meantime. In the present study, we therefore re-evaluated the use of G-CSF after ASCT
Ann Hematol Oncol - Volume 4 Issue 5 - 2017

ISSN : 2375-7965 | www.austinpublishing group.com

Pabst et al. (C) All rights are reserved
Citation: Oberson JS, Novak U, Taleghani BM, Baerlocher GM, Seipel K, Mueller BU, et al. Revisiting G-CSF Support for Hematologic Recovery after Autologous Transplantation in AML Patients. Ann Hematol Oncol. 2017; 4(5): 1148. 
Table 1: Hematologic recovery and infectious complications after autologous stem cell transplantation in AML patients in first complete remission.

\begin{tabular}{|c|c|c|c|c|}
\hline & all & +G-CSF & -G-CSF & $\mathbf{P}$ \\
\hline$n$ & 115 & 103 & 12 & \\
\hline $\mathrm{CD} 34^{+} \times 10^{6} \mathrm{cells} / \mathrm{kg}$ given, median & 4.4 & 4.4 & 3.9 & 0.2565 \\
\hline $\begin{array}{l}\text { Platelet recovery >20G/L, days, } \\
\text { median }\end{array}$ & 16 & 16 & 16 & 0.7017 \\
\hline$>50 G / L$, days & 40.5 & 38.5 & 42.5 & 0.9145 \\
\hline$>100 \mathrm{G} / \mathrm{L}$, days & 92 & 88 & 106 & 0.6155 \\
\hline Platelet transfusion, $n$, median & 3 & 3 & 2 & 0.9563 \\
\hline Neutrophil recovery $>0.5 \mathrm{G} / \mathrm{L}$, days & 12 & 12 & 16 & $.0001^{* *}$ \\
\hline$>1.0 \mathrm{G} / \mathrm{L}$, days & 13 & 13 & 18 & $.0020^{* *}$ \\
\hline Red cell transfusions, $\mathrm{n}$, median & 2 & 2 & 2 & 0.7351 \\
\hline Febrile episode (at least once $\geq 38.5$ & 88.6 & 87.7 & 100 & 0.1932 \\
\hline Bacteremia, \% ${ }^{\circ}$ & 41.2 & 38.3 & 66.7 & 0.0654 \\
\hline Patients with gram pos, $\mathrm{n}(\%)^{\mathrm{i}}$ & $31(28.8)$ & $27(26.2)$ & $4(33.3)$ & 0.6005 \\
\hline Patients with gram neg, $n(\%)$ ii & $23(21.2)$ & $19(18.4)$ & $4(33.3)$ & 0.2244 \\
\hline Fungal, n (\%) $)^{\mathrm{iii}}$ & $14(12.3)^{i}$ & $13(12.7)$ & $1(9.1)$ & 0.6686 \\
\hline Viral, n (\%) & $10(8.8)$ & $10(9.7)$ & $0(0)$ & 0.2607 \\
\hline Hospitalization, days, median & 23 & 23 & 25 & 0.0603 \\
\hline Hospitalization $\geq 30$ days, $n$ & 17 & 16 & 1 & 0.5078 \\
\hline
\end{tabular}

'Some patients had several germs identified; 'Coagulase-negative Staphylococcus ( $\mathrm{n}=18+\mathrm{G}-\mathrm{CSF} / 2$-G-CSF patients); Staphylococcus aureus ( $\mathrm{n}=2 / 0)$; Streptococcus viridans $(n=4 / 1)$; Clostridium perfringens $(n=1 / 0)$; Staphylococcus lugdunensis $(n=1 / 0)$; Corynebacterium sp. $(n=2 / 0)$; Bacillus cereus $(n=2 / 1)$; "E. coli $(n=9 / 2)$; Klebsiella pneumoniae $(n=6 / 1)$; Enterobacter cloacae $(n=3 / 0)$; Proteus mirabilis $(n=2 / 0)$; ESBL E. coli $(n=1 / 0)$; Klebsiella oxytoca $(n=2 / 1)$; Moraxella catarrhalis $(n=1 / 0)$; iiioralnm candidiasis $(n=5 / 0)$; hepatosplenic candidiasis infection $(n=3 / 0)$; esophageal candidiasis $(n=2 / 0)$; genital candidiasis $(n=1(1)$; probable invasive pulmonary aspergillosis sp. $(n=1 / 0)$; ${ }^{i}$ Herpes Zoster $(n=11 / 0)$; Herpes simplex stomatitis $(n=6 / 0)$; CMV reactivation $(n=1 / 0)$.

in AML patients comparing two consecutive cohorts of patients with G-CSF support (+G-CSF; January 2004 until June 1, 2015) after ASCT versus without G-CSF (-G-CSF; from June 2015 until November 2016).

\section{Patients and Methods}

\section{Patients}

In this single center retrospective analysis, we investigated consecutive AML patients in CR1 receiving HDCT with ASCT after two cycles of induction chemotherapy at the University Hospital of Bern, Switzerland between January 2004 and November 2016. Patients underwent HDCT with ASCT if the genomic risk assessment indicated good-risk, intermediate-risk (in the absence of an HLA-identical sibling donor), or bad/very bad risk AML (in the absence of a sibling and an unrelated matched donor). Risk assessment was performed according to the European Leukemia Net (ELN) classification. The clinical characteristics of the patients are summarized in Table 1. This study was approved by the local ethics committee of Bern, Switzerland (decision number \#220/15).

\section{Treatment}

The patients were uniformly treated within subsequent protocols of the Dutch-Belgian Hemato-Oncology Cooperative Group (HOVON) / Swiss Group for Clinical Research (SAKK), including the
SAKK/HOVON-42, -92, -102 and -132 protocols. Patients received cytarabine $200 \mathrm{mg} / \mathrm{m}^{2}$ on days $1-7$ and idarubicin $12 \mathrm{mg} / \mathrm{m}^{2}$ days $1-3$ in induction cycle 1 ; and cytarabine $1000 \mathrm{mg} / \mathrm{m}^{2} / \mathrm{q} 12 \mathrm{~h}$ days $1-6$ and amsacrin $120 \mathrm{mg} / \mathrm{m}^{2}$ days $1-3$ were given in cycle 2 , which was also used for subsequent mobilization of peripheral blood stem and progenitor cells (PBSC). G-CSF stimulation at $10 \mu \mathrm{g} / \mathrm{kg}$ body weight (b.w.) was initiated on the first day of cycle 2 when neutrophils increased above $0.5 \mathrm{G} / \mathrm{L}$. Stem cell collection was performed on the first day when the number of peripheral circulating CD34+ cells exceeded 20,000/ $\mathrm{ml}$. For HDCT, patients received oral busulfan $4 \mathrm{mg} / \mathrm{kg}$ b.w./day on days -6 until -3 , and intravenous cyclophosphamide $60 \mathrm{mg} / \mathrm{kg} / \mathrm{day}$ on days -2 and -1 . A minimum of $2.0 \times 10^{\wedge} 6 \mathrm{CD} 34+$ cells $/ \mathrm{kg}$ b.w. was transfused at the day of autologous stem cell transplantation (day 0 ).

Before June 2015, patients received 30 Mio U (<78kg b.w.) or 48 Mio U ( $\geq 78 \mathrm{~kg}$ ) of G-CSF as a once daily s.c. injection from days +6 to +12 after ASCT. In June 2015, we changed our strategy and committed to reevaluate it after 12 treated patients; thus, patients after June 2015 received no G-CSF for hematologic recovery after ASCT. Patients in the two cohorts were evaluated for hematologic recovery, number of platelet and erythrocyte transfusions, febrile episodes and bacteremias, duration of hospitalization and costs.

All patients undergoing HDCT with ASCT routinely had antiviral (oral acyclovir $500 \mathrm{mg}$ twice daily) and antifungal prophylaxis (oral fluconazole $400 \mathrm{mg}$ once weekly), and oral sulfamethoxazol/ trimethoprim $800 / 160 \mathrm{mg}$ three times per week. No routine antibiotic prophylaxis was given. Hyperuricemia prophylaxis with $300 \mathrm{mg}$ oral allopurinol was applied on days -7 to -1 . Patients received platelet and red cell transfusions when platelets fell $<10 \mathrm{G} / \mathrm{L}$ or if clinically indicated (bleeding, need for interventions), and hemoglobin was $<80 \mathrm{~g} / \mathrm{L}$, respectively. Patients were hospitalized for the entire HDCT/ ASCT procedure and dismissed after hematologic recovery and adequate physical reconditioning.

\section{Definitions}

Overall survival was defined as the time from ASCT until death from any cause or until the last follow-up. Disease-free survival was defined as the time from the date of ASCT until the first progression of AML or the last follow-up, whichever occurred first. Follow-up was defined as the time from ASCT until the last documented patient visit in our department. Patients still alive or lost to follow-up were censored at the last date when they were known to be alive.

\section{Statistical analysis}

Survival curves were analyzed using the log-rank (MantelCox) test. The unpaired t-test was applied to compare continuous variables. The Mann- Whitney test was used to compare the median of parameters. In order to compare specific parameters between the two groups, the two proportion test (based on the N-1 chi-square test) was applied. All P-values were two-sided, indicated with the $\mathrm{P}$, and not adjusted for multiple testing, unless explicitly indicated. A P-value below 0.05 was considered significant. The cut-off date for this analysis was November 1, 2016. For the statistical analysis, GraphPad Prism Version 5.0c (GraphPad Software Inc., La Jolla, CA, USA) was used.

\section{Results}

\section{Hematologic recovery}

From January 2004 to June 2015, 103 AML patients in CR1 were 

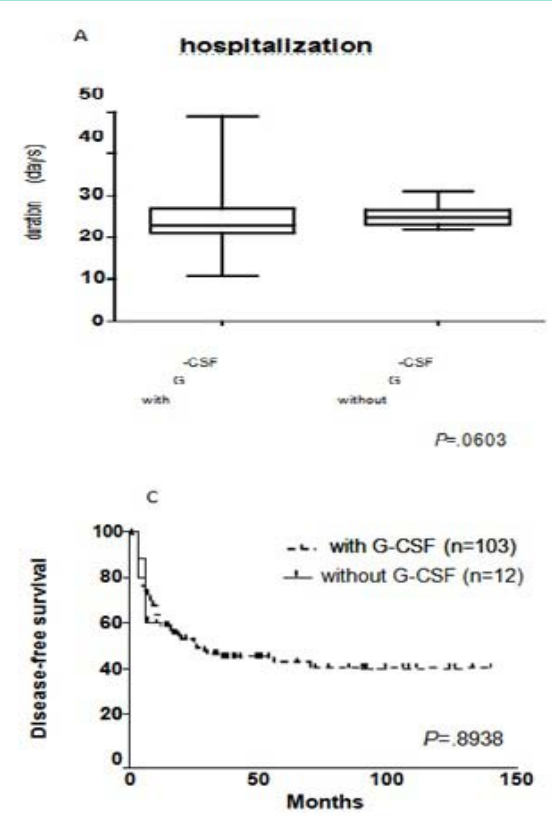

B Duration (days) ANC $>0.5 \mathrm{G} / \mathrm{L}$
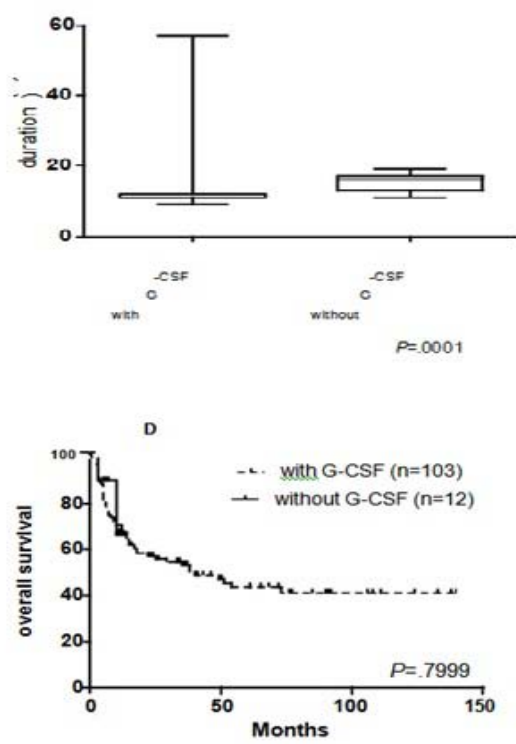

Figure 1: Box plot presentations of (A) duration of hospitalization in days, and (B) duration until recovery of neutrophils above $0.5 \times 10^{9} / \mathrm{L}$. Boxes comprise the range from the $25^{\text {th }}$ to the $75^{\text {th }}$ percentile, with the middle bar indicating the median value. Whiskers represent values below the $25^{\text {th }}$ percentile to the minimum value and above the $75^{\text {th }}$ percentile to the maximum value, respectively. Kaplan-Meyer curves present $(C)$ disease-free survival and (D) overall survival of $A M L$ patients after two cycles of induction treatment calculated from the day of ASCT.

uniformly treated with G-CSF after ASCT, whereas 12 additional consecutive AML patients underwent HDCT with ASCT after June 2015 without G-CSF support. We observed that the median time from ASCT (day 0) until neutrophil recovery $>0.5 \mathrm{G} / \mathrm{L}$ was 12 days (range 9-57 days) for +G-CSF patients and 16 days (range 11-19 days) without G-CSF $(P=.0001)$ (Figure 1A). Similarly, the median time until neutrophil recovery $>1.0 \mathrm{G} / 1$ was 13 days (range 10-57 days) for $+\mathrm{G}$-CSF patients and 18 days (range 11-54 days) without G-CSF $(P=.0020)$ (Supplementary Figure $1 \mathrm{~A})$, whereas no differences were observed for platelet recovery $>20 \mathrm{G} / \mathrm{L}(P=.7017)$ (Supplementary Figure $1 \mathrm{~B}),>50 \mathrm{G} / \mathrm{L}(P=.9145)$, or $>100 \mathrm{G} / \mathrm{L}(P=.6155)$. Also, the median number of platelet transfusions (three versus two units; $P=.9563$ ) and of red blood cell transfusions (two units in both cohorts; $P=.7351)$ were comparable.

\section{Infectious complications}

During the neutropenic phase $(<0.5 \mathrm{G} / \mathrm{L})$, at least one febrile episode ( $\mathrm{T}$ above $38.5^{\circ} \mathrm{C}$ ) occurred in $87.7 \%$ of the $+\mathrm{G}$-CSF patients and in all -G-CSF patients $(P=.1932)$. Blood cultures documented bacteremia in $66.7 \%$ of the -G-CSF patients and in $38.3 \%$ of the $+\mathrm{G}-\mathrm{CSF}$ patients $(P=.0654)$. The predominant germs causing bacteremia were coagulase-negative staphylococci (18 patients), E. coli (nine patients), and Klebsiella spp. (six patients) (Supplementary Table 1). Finally, 2 of $103(2 \%)+$ G-CSF patients and 1 of $12(8 \%)$ -G-CSF patient needed to be transiently treated in the intensive care unit during the neutropenic phase following ASCT: one patient had a transient confusional state during a febrile episode, and two patients needed intravenous pharmacologic support for hypotension during sepsis.

\section{Duration of hospitalization}

The median duration of hospitalization was two days shorter in + G-CSF patients (25 days versus 23 days; $P=.0603$; (Figure 1B). The duration of hospitalization ranged between 11 and 49 days for +G-CSF patients, and between 22 and 31 days for -G-CSF patients. Hospitalization exceeded 30 days in 17 patients $(16+$ G-CSF patients and one-G-CSF patient) due to delayed hematological recovery with ongoing transfusion needs (ten patients), infections (five patients), and cholecystitis (two patients). The rehospitalization rate was $12.2 \%$ for $+\mathrm{G}-\mathrm{CSF}$ patients and $0 \%$ for $-\mathrm{G}-\mathrm{CSF}$ patients $(P=.2)$.

\section{Costs}

We also assessed the financial burden associated with the use of G-CSF after ASCT. Current total costs for seven daily applications of 48 Mio U G-CSF are 194.60 CHF, and 133.00 CHF for 30 Mio U daily, respectively. In contrast, costs for an additional day of hospitalization for ASCT in AML patients at our hospital currently account for 1750 CHF according to the current version of the diagnosis-related index of the national Swiss in-patient reimbursement system, resulting in decreased total costs for an average $+\mathrm{G}-\mathrm{CSF}$ patient of $3305.40 \mathrm{CHF}$ (48 Mio U), and 3367.00 CHF (30 Mio U), respectively, based on our observation that -G-CSF patients were hospitalized two days longer.

\section{Survival and relapse rates}

Finally, we compared disease-free (DFS) and overall survival (OS) of +G-CSF and -G-CSF patients (Figure 1C, 1D) and depicting DFS and OS at 12 months after ASCT in Supplementary Figure 1C, 1D. Due to the chronologic order of the cohorts, the median followup was different, with 41.2 months for the +G-CSF patients and 5.3 months for the -G-CSF patients, respectively. So far, $55+\mathrm{G}-\mathrm{CSF}$ patients $(53.4 \%)$ relapsed and three of 12 -G-CSF patients (25\%) $(P=.0637$; Supplementary Table S2). Relapse in the three -G-CSF patients occurred after a median of five months after ASCT, whereas the median duration from ASCT to relapse was 15 months in $+\mathrm{G}-\mathrm{CSF}$ 
patients $(P=.1010) .48$ of the relapsing patients were treated with salvage chemotherapy, three patients had best supportive care, and seven patients received palliative chemotherapy. 26 patients achieved a second complete remission, and 20 (all in the +G-CSF cohort) relapsing patients ultimately underwent allogeneic transplantation in second remission. Finally, among patients with a minimum followup of at least 12 months after ASCT, $64.1 \%$ of + G-CSF patients and $61.7 \%$ of -G-CSF patients were in ongoing first remission.

So far, 55 patients died including $53+\mathrm{G}$-CSF patients (51.5\%) and two -G-CSF patients $(16.7 \% ; P=.0230) .50$ of the 55 deaths were related to AML progression (46 patients) or to allogeneic transplantation (four patients), whereas the remaining five patients died of secondary cancer (breast cancer, one patient), suicide (one patient), epileptic seizure (one patient), herniation of a incisional hernia (one patient), and for unknown causes (one patient). 32 of 103 (31.1\%) +G-CSF patients and two (16.7\%) of 12 -G-CSF patients died within one year after ASCT.

\section{Discussion}

This study has the limitations of a single-center retrospective analysis, including the small sample size of the -G-CSF group, and any findings using this approach ideally must be verified by a prospective comparison. However, it is highly unlikely that any collaborative study group will ever run an adequately powered randomized trial comparing the use of G-CSF versus no G-CSF after ASCT in AML. Consequently, decision making in clinical routine will rely on reports such as ours suggesting that administering G-CSF for hematologic recovery after ASCT in AML in CR1 is associated with a shorter duration of neutropenia, fewer febrile episodes and fewer bacteremias, as well as a shorter duration of hospitalization and, thus, reduced overall costs. Similar to the data on the use of G-CSF for hematologic recovery during induction treatment, our study also suggests no differences in survival rates between AML patients treated with and without G-CSF after ASCT. Our data also point to the pitfalls inherent with adapting guidelines driven by increasing economic pressure as evidenced by the collapsing prizes for G-CSF, and they advocate for a comprehensive view in decision-making when treating AML patients.

\section{Acknowledgement}

The authors wish to thank the stem cell coordinating team, the stem cell data management team, and the members of the stem cell collection unit and of the stem cell processing unit associated with the stem cell program at the University Hospital Berne for providing data used in this analysis. Also, the authors wish to thank all staff members at the University Hospital Berne involved in the care of the patients reported in this study.

\section{Author's Contributions}

J.O. performed research, analyzed data and wrote the paper; K.S. and B.U.M. analyzed data, reviewed the manuscript and were involved in the final writing of the paper; B.M.T., G.M.B., U.N., K.L., Y.B., and S.Z. Contributed relevant data, reviewed the manuscript and were involved in the final writing of the paper; and T.P. designed research, analyzed data and wrote the paper.

\section{Funding}

Supported by a grant of the Swiss Cancer League KFS-3795-022016 to T.P.

\section{References}

1. Burnett AK, Russell NH, Hills RK, Kell J, Cavenagh J, Kjeldsen L, et al. A randomized comparison of daunorubicin $90 \mathrm{mg} / \mathrm{m}^{2}$ vs $60 \mathrm{mg} / \mathrm{m}^{2}$ in $\mathrm{AML}$ induction: results from the UK NCRI AML17 trial in 1206 patients. Blood. 2015; 125: 3878-3885.

2. Dohner H, Estey EH, Amadori S, Appelbaum FR, Büchner T, Burnett AK, et al. Diagnosis and management of acute myeloid leukemia in adults: recommendations from an international expert panel, on behalf of the European Leukemia Net. Blood. 2010; 115: 453-474

3. Cornelissen JJ, Versluis J, Passweg JR, van Putten WL, Manz MG, Maertens $\mathrm{J}$, et al. Comparative therapeutic value of post-remission approaches in patients with acute myeloid leukemia aged 40-60 years. Leukemia. 2015; 29 : 1041-1050.

4. O'Donnell MR, Tallman MS, Abboud CN, Altman JK, Appelbaum FR, Arber DA, et al. Acute myeloid leukemia. J Natl Compr Cancer Netw. 2013; 11: 1047-1055.

5. Zuckerman T, Beyar-Katz O, Rowe JM. Should autotransplantation in acute myeloid leukemia in first complete remission be revisited? Curr Opin Hematol. 2016; 23: 88-94.

6. Gorin NC, Giebel S, Labopin M, Savani BN, Mohty M, Nagler A. Autologous stem cell transplantation for adult acute leukemia in 2015: time to rethink? Present status and future prospects. Bone Marrow Transplant. 2015; 50: 1495-1502.

7. Vellenga E, van Putten W, Ossenkoppele GJ, Verdonck LF, Theobald M Cornelissen $\mathrm{JJ}$, et al. Autologous peripheral blood stem cell transplantation for acute myeloid leukemia. Blood. 2011; 118: 6037-6042.

8. Wang J, Ouyang J, Zhou R, Chen B, Yang Y. Autologous hematopoietic stem cell transplantation for acute myeloid leukemia in first complete remission: a meta-analysis of randomized trials. Acta Haematol. 2010; 124: 61-71.

9. Heuser M, Zapf A, Morgan M, Krauter J, Ganser A. Myeloid growth factors in acute myeloid leukemia: systematic review of randomized controlled trials. Ann Hematol. 2011; 90: 273-281.

10. Sierra J, Szer J, Kassis J, Herrmann R, Lazzarino M, Thomas X, et al. A single dose of pegfilgrastim compared with daily filgrastim for supporting neutrophil recovery in patients treated for low-to-intermediate risk acute myeloid leukemia: results from a randomized, double-blind, phase 2 trial. BMC Cancer. 2008; 8: 195-201.

11. Heil G, Hoelzer D, Sanz MA, Lechner K, Noens L, Szer J, et al. Long-term survival data from a phase 3 study of Filgrastim as an adjunct to chemotherapy in adults with de novo acute myeloid leukemia. Leukemia. 2006; 20: 404-409.

12. Harousseau JL, Witz B, Lioure B, Hunault-Berger M, Desablens B, Delain $M$, et al. Granulocyte colony-stimulating factor after intensive consolidation chemotherapy in acute myeloid leukemia: results of a randomized trial of the Groupe Ouest-Est Leucémies Aigues Myeloblastiques. J Clin Oncol. 2000; 18: $780-787$.

13. Heil G, Hoelzer D, Sanz MA, Lechner K, Liu Yin JA, Papa G, et al. A randomized, double-blind, placebo-controlled, phase III study of filgrastim in remission induction and consolidation therapy for adults with de novo acute myeloid leukemia. The International Acute Myeloid Leukemia Study Group. Blood. 1997; 90: 4710-4718.

14. Pabst T, Vellenga E, van Putten W, Schouten HC, Graux C, Vekemans MC et al. Favorable effect of priming with granulocyte colony-stimulating factor in remission induction of acute myeloid leukemia restricted to dose escalation of cytarabine. Blood. 2012; 119: 5367-5373. 